\title{
Structural basis of CK2 regulation by autoinhibitory oligomerization
}

Graziano Lolli*1,2 \& Roberto Battistutta*1,2

${ }^{1}$ Department of Chemical Sciences, University of Padua, via Marzolo 1, 35131 Padua, Italy.

${ }^{2}$ Venetian Institute for Molecular Medicine (VIMM), via Orus 2, 35129 Padua, Italy.

* Corresponding authors. Department of Chemical Sciences, via Marzolo 1, 35131 Padua ITALY.

Tel. +39.049.7923236, Fax: +39.049.7923250, e-mail: graziano.lolli@unipd.it.

roberto.battistutta@unipd.it.

\section{Keywords:}

Crystal structure; symmetric CK2 holoenzyme; asymmetric CK2 holoenzyme; trimeric CK2 holoenzyme; oligomerization; filaments; activity regulation; autoinhibition; crystal contacts; $\alpha$ subunit; $\beta$-subunit; $\alpha_{2} \beta_{2}$ tetramer; $\alpha / \beta$ interfaces; CK2 $\beta$ acidic loop; CK2 $\alpha p+1$ loop; CK2 $\beta$ Cterminal tail; electrostatic interactions; polyamines; autophosphorylation. 


\begin{abstract}
The mechanism of regulation of CK2 differs from those common to other eukaryotic protein kinases and is not entirely established yet. Nowadays several crystal structures of the tetrameric $\alpha_{2} \beta_{2}$ holoenzyme are available, supporting a structural model of an autoinhibitory regulation by oligomerization proposed several years before on the basis of biochemical, biophysical and functional data. Monoclinic crystal forms of the holoenzyme reveal the symmetric architecture of the "free" isolated active tetramers. The dimension and the nature of the $\alpha / \beta$ interfaces configure the symmetric holoenzyme as a strong complex that does not spontaneously dissociate in solution, in accordance with the low dissociation constant $(\approx 4 \mathrm{nM})$. Hexagonal crystal forms of the CK2 holoenzyme show an asymmetric arrangement of the two $\alpha$ catalytic subunits around the obligate $\beta_{2}$ regulatory subunits. These asymmetric $\alpha_{2} \beta_{2}$ tetramers are organized in trimeric rings and filaments that correspond to inactive forms of the enzyme, whereby the $\beta$-subunit plays an essential role in the formation of inactive polymeric assemblies. The derived structural model of (down)-regulation by aggregation contributes to the interpretation of many available biochemical and functional data, although awaits for a more comprehensive validation at cellular level. These findings pave the way for the design of novel strategies aimed at the modulation of the CK2 activity.
\end{abstract}


The first crystallographic structure of the CK2 holoenzyme was determined in 2001 (PDB 1JWH) [1] joining those already known of the isolated $\alpha$ and $\beta$ subunits [2-3], a great achievement finally solving the puzzling CK2 quaternary arrangement. CK2 tetramer has a butterfly shape with a central $\beta$-dimer and the two $\alpha$ subunits one on each side and not in contact between them (Figure 1a). This was also the first structure including the full-length $\beta$ subunit that can be divided into a body and a C-terminal tail, the last one not included in previous structures. CK2 $\beta$ C-terminal tail has no contact with the body of its own chain but binds both the other CK2 $\beta$ monomer and one of the $\alpha$ subunits (Figure 1a). This tail contributes significantly to the stability of the $\beta$-dimer. The $\beta / \beta$ interface increases from $543 \AA^{2}$ in the structure of the isolated and truncated $\beta$-dimer to $1766 \AA^{2}$ in the structure of the holoenzyme, in agreement with the obligate nature of the $\beta$-dimer. The authors also highlighted that this increase in the interface requires the CK2 $\beta$ tail in a specific conformation, which is stabilised by the involvement of the same tail in large contacts with the $\alpha$ subunits and hence depends on the existence of the complete tetramer. The CK2 $\beta$ tail has then a synergistic character, stabilizing both the $\beta / \beta$ and the $\alpha / \beta$ contacts. At the same time, the authors pointed out that this region was not well defined in the electron density and then the very C-terminal amino acids (from Asn206 to Arg215) were left out from the final model while in the preceding region (from Phe190 to Ser205) the conformations of peptide groups and side chains remain questionable.

Each $\alpha$ subunit binds both subunits of the $\beta$-dimer, the body of one chain and the tail of the other (Figure 1a), this last interaction exploring a larger interface $\left(491 \AA^{2}\right.$ vs $\left.336 \AA^{2}\right)$ and being considered the major determinant of the $\alpha / \beta$ stability. The structure of the $\alpha$ catalytic subunit is not significantly affected by the binding of the $\beta$-dimer, maintaining its active conformation and confirming previous observations that $\mathrm{CK} 2 \beta$ is an environment- and substrate-dependent modulator of CK2 $\alpha$ activity rather than an on/off switch. It was also noted that the tetramer is asymmetric with a relative rotation of $16.4^{\circ}$ at the two $\alpha / \beta$ interfaces for the two halves of the tetramer (Figure 1a). As a consequence, the two $\alpha / \beta$ interfaces are different (Figure $1 \mathrm{~b}$ ) and this was interpreted as an 
intersubunit flexibility. In the basis of such observation, together with an average size for the $\alpha / \beta$ interface of $832 \AA^{2}$, authors proposed that the CK2 tetramer is a transient heterocomplex.

Finally, in the same paper it was pointed out that the inhibitory effect of the CK2 $\beta$ acidic loop (Asp55-Asp64) observed in previous works [4] cannot be exerted intramolecularly since the distance between this and the closest CK2 $\alpha$ basic cluster (the potential substrate binding site) was $>30 \AA$.

A few years later, the same authors analysed the crystal packing in the previous structure showing that the contact between the $\beta$-acidic loop and the $\alpha$-basic cluster was indeed present, but between different tetramers [5]. They identified a new CK2 organisation, composed by selfconsistent ring-like trimers of tetramers, where the core of the interaction between different tetramers in $\left(\alpha_{2} \beta_{2}\right)_{3}$ is formed by the interface between the $\beta$-acidic loop and the $\alpha p+1$ loop (Arg191-Lys 198) at the entrance of the $\alpha$ active site (Figure 1c). As a consequence, three out of six catalytic subunits of the trimeric ensemble are blocked by the $\beta$ acidic loop.

This organisation in trimers of tetramers was able to explain a number of previous observations not easily interpretable solely on the basis of the tetrameric structure. Mutations in the $\beta$-acidic loop or in the $\alpha p+1$ loop generate hyperactive CK2 holoenzyme $[4,6]$, since trimers of tetramers cannot form anymore. Polyamines like spermine activate CK2 [7-9] by interacting with the CK2 $\beta$ acidic loop and then disrupting the trimers of tetramers. The CK2 holoenzyme exhibits maximum activity at about $300 \mathrm{mM} \mathrm{NaCl}$ or $\mathrm{KCl}$, a property of the holoenzyme and not of the isolated $\mathrm{CK} 2 \alpha[10]$. The holoenzyme also needs a minimal $\mathrm{NaCl}$ concentration of $250 \mathrm{mM} \mathrm{NaCl}$ to bind to phosphocellulose, while it aggregates under low salt conditions [11]. Since the interaction between the $\beta$ acidic loop and the $\alpha p+1$ loop is electrostatic in nature, it is strongly affected by ionic strength. Finally CK2 holoenzyme has been observed in different ring-like and linear polymeric forms connected via reversible equilibria affected by ionic strength and spermine [12-13]. 
In 2012 we presented a new structure of the holoenzyme (4DGL) crystallized in the same space group and unit cell of the previous $1 \mathrm{JWH}$ structure, but with higher overall quality [14]. While confirming may features of the 1JWH structure, the 4DGL structure shows significant improvements in important regions such as the $\alpha / \beta$ interface and the $\mathrm{C}$-terminal tail of the $\beta$ subunit. In particular, $\beta$-Phe190 leans on the outer surface of the a N-terminal lobe in 1JWH, while in 4DGL it inserts deeply into a hydrophobic cavity in the $\alpha$ subunit, known as the secondary or allosteric binding site (Figures $2 \mathrm{a}$ and $2 \mathrm{~b}$ ). The $\alpha / \beta$ interaction is then significantly strengthened in accordance with the almost complete abrogation of the binding for the mutant $\beta$-Phe190Ala [15]. Also the $\beta$ C-terminus was build differently with an upstream shift in sequence of two amino acids and extended the $\alpha \mathrm{G}$ helix from residues 196-200 in 1JWH to residues 194-207 in 4DGL (Figure $2 b$ ). This again contributes to refine the $\alpha / \beta$ interface to a better energetic profile (interface increased from $832 \AA^{2}$ in $1 \mathrm{JWH}$ to $1010 \AA^{2}$ in 4DGL (Figure $2 \mathrm{c}$ ). Finally the $\beta \mathrm{C}$-terminus was build to the last Arg215 amino acid.

As already noted by Niefind and Issinger [5], the acidic loop of the $\beta$-subunit from one tetramer is in contact with the $p+1$ loop of the $\alpha$ subunit from another tetramer (Figure 3a). The interface area is $651 \AA^{2}$, larger than in $1 \mathrm{JWH}\left(538 \AA^{2}\right)$ and greater than normal crystallographic contacts, which have a mean interface of $285 \AA^{2}$ and rarely exceed $500 \AA^{2}$ [16]. This interaction is essential for the organization of circular trimers of tetramers as previously observed (Figure 1c), with a positive cooperative effect for their formation and a large interface of $1953 \AA^{2}\left(651 \AA^{2} \times 3\right)$.

The newly traced C-terminus of CK2 $\beta$ is instead responsible of a different inter-tetrameric interaction. This region protrudes from one tetramer and contacts the C-terminal lobe of an $\alpha$ subunit of a neighbouring tetramer ending in its ATP pocket (Figures 3a and 3b). The interface is large $\left(753 \AA^{2}\right)$ and is complemented by the interaction between the N-terminal lobe and the Cterminal lobe of two $\alpha$ chains from the same two tetramers. The overall interface of $1254 \AA^{2}$ is then quite extended and again much larger than a normal crystallographic contact. It shows a number of polar contacts, which again makes this interaction susceptible to ionic strength changes. This new 
interaction is fundamental for a piling organization of trimers of tetramers (Figures 3c) and the newly identified polymeric form can represent the filamentous CK2 aggregates described in literature [12-13]. Moreover, the $\beta$ C-terminus inserts into the ATP binding pocket of a catalytic subunits, competing with ATP and stabilizing a non-productive conformation of the $\alpha$ catalytic residues (Figure 3b). This polymeric form is then expected to be completely inactivated by the combination of the interactions of the $\alpha$ subunits with the acidic loops and C-termini of $\beta$ subunits (Figure 3a), and by steric hindrance.

This new structure and the hypothesized organization in trimers of tetramers and subsequently in filaments allow expanding the experimental data previously reported in support of the trimeric organization by Niefind and Issinger [5]. FRET experiments revealed the existence in vivo of two subpopulations of "fast" and "slow moving" CK2 and showed that different CK2 $\beta$-dimers can be in close molecular proximity [17]. The down-regulating effect proposed for the CK2 $\beta$ C-terminal tail is in accordance with other in vivo observations. CK2 activity is increased in prostate cancer cells by CDK1 phosphorylation of $\beta$-Ser209 [18] that engages $\alpha$-Glu230 in the filamentous organization, which will then be weakened by the phosphorylation event. Phosphorylation of $\beta$ Thr213 by Chk1 is observed in the free $\beta$ subunits but not in the tetrameric holoenzyme [19], where the residue is masked in the filamentous oligomers. The down-regulatory polymerization mechanism mediated by the $\beta$ subunits is also supported by the observation that transfection of HeLa cells with a degradation-resistant CK2 $\beta$ strongly inhibits proliferation and counteracts promotion of proliferation exerted by the isolated CK2 $\alpha[20]$.

It is well known that CK2 autophosphorylates on residues Ser2 and Ser3 of the $\beta$-chain [21]. As already observed by Niefind and Issinger [5] and further discussed in [14], the $\beta \mathrm{N}$-terminus is close to an $\alpha$ catalytic site in the trimeric organization, so that one tetramer could phosphorylate the neighbouring one (Figure 3a). Observations in favour of this trans-autophosphorylation mechanism inside the trimeric organization are the following: autophosphorylation (a) is abolished at high ionic strength [22], (b) is strongly inhibited by neutralization of residues in the $\beta$-acidic loop [23], (c) 
although being in trans, it shows an intramolecular kinetic [24], (d) no autophosphorylation occurs in the holoenzyme with the $\alpha^{\prime}$ paralog, a holoenzyme that does not form oligomers [25]. We also proposed that the $\beta \mathrm{N}$-terminus, once phosphorylated, could engage the nearby $\alpha \mathrm{C}$ basic cluster of the $\alpha$ subunit from the neighbouring tetramer, further stabilizing the ring-like organization. Autophosphorylation is (e) extensively observed in cells [21], (f) stabilizing the enzyme in vivo [26] and (g) having an inhibitory effect, since treatment of CK2 with phosphatases increases its activity [27].

\section{The symmetric $\alpha_{2} \beta_{2}$ holoenzyme crystal structures}

Very recently, we added to the above picture three additional crystal structures of the $\alpha_{2} \beta_{2}$ holoenzyme [28]. In both the 1JWH and 4DGL structures, the $\alpha_{2} \beta_{2}$ tetramer is asymmetric. Notably, in the new structures (4MD7, 4MD8 and 4MD9), obtained in monoclinic (rather than hexagonal) space groups, the tetramer is symmetric (Figure 4a). This reveals that the asymmetry observed in the previous structures is not an intrinsic structural property of the monomeric holoenzyme, but rather is generated by the incorporation of tetramers into trimeric rings, whose assembly is not compatible with the symmetric architecture (Figure 4b). The analysis of the crystal packing of the monoclinic form of the holoenzyme does not seem to support the possibility of stable oligomeric or filamentous forms. Unlike in the hexagonal lattice, where discrete trimeric rings of tetramers are clearly recognizable, in the monoclinic form all contacts between different tetramers have a pure crystallographic nature. Indeed there is one interaction between the acidic loop of the $\beta$-subunit and the basic clusters of the $\alpha$-subunit of a symmetric tetramer with a significant interface area (around $750 \AA^{2}$ ). However, this contact is not responsible for the formation of any closed, self-consistent, oligomeric form of tetramers, nor of a filament of tetramers. Rather it is responsible for the propagation of two parallel layers of tetramers in two dimensions for the entire crystal lattice (i.e. infinitively), giving raise to a sort of infinite two-dimensional bilayer of tetramers. Hence, it has the characteristic of a crystal lattice contact, making highly improbable, in our opinion, the existence of 
stable discrete oligomeric or filamentous forms based on this interaction. We then concluded that the new symmetric structures represent the organization of the "free", active, monomeric CK2 holoenzyme, the previous $1 \mathrm{JWH}$ and $4 \mathrm{DGL}$ showing instead the structure of inactive trimers of tetramers. However, it has to be noted that some of the inter-tetrameric interactions typical of the monoclinic crystal packing, observed also for the CK2 ${ }^{\text {andante }}$ holoenzyme mutant [29], have been postulated to be at the basis of other possible (linear) assemblies with physiological significance $[30]$.

The comparison of symmetric and asymmetric tetramers in terms of their $\alpha / \beta$ interfaces and then of their stability is of particular relevance since the possibility that the CK2 holoenzyme coexists in equilibrium with its free constituent subunits can represent a regulatory mechanism for its biological function. In $1 \mathrm{JWH}$, the two $\alpha / \beta$ interfaces are significantly different in size, one being larger $\left(960 \AA^{2}\right)$ and the other significantly smaller $\left(771 \AA^{2}\right)$ [1] (Figure 1b). This suggested that the CK2 holoenzyme might be a transient heterocomplex, spontaneously dissociating in vivo. This seems in contrast with the well-known elevated stability in vitro of the complex $\left(\mathrm{K}_{\mathrm{D}} \approx 4 \mathrm{nM}\right)[31$ 32]. In 4DGL, the better-traced $\beta$ C-terminal tail generated larger interfaces with similar sizes (1009 and $1011 \AA^{2}$ ); however, due to the asymmetric assembly, the two interfaces are different in nature with different residues involved [14] (Figure 2b).

The new symmetric structures have very similar $\alpha / \beta$ interfaces both in size (1099 and $\left.1073 \AA^{2}\right)$ and chemical nature (Figure 4c). This was also confirmed by PISA ('Protein interfaces, surfaces and assemblies' service at the European Bioinformatics Institute [33]) that predicted a dissociation pattern in $2 \alpha+\beta_{2}$ from monoclinic structures, but in $\alpha \beta_{2}+\alpha$ for both 1 JWH and 4DGL as a consequence of their asymmetry. The interface size is the most commonly used parameter to infer the stability of a complex; indeed also its chemical and sterical nature (hydrophobicity and complementarity) needs to be considered [34]. Evaluating a number of parameters (reported in Table 1), we proposed that the CK2 holoenzyme configures as a non-obligate permanent heterocomplex. In obligate complexes, the constitutive protomers are not stable in the isolated form 
and they simultaneously fold and bind to their partner [35-36], as in the case of the CK2 $\beta$ dimer. Obligate complexes have interface areas generally much larger than $1000 \AA^{2}$ [37]. The CK2 holoenzyme is obviously a non-obligate complex. Permanent protein-protein interactions are strong, with nanomolar or subnanomolar dissociation constants, and irreversible, normally only perturbed by proteolysis [35-36]. Transient complexes can instead dissociate in their constituent subunits and can be distinguished in weak or strong [35-36]. In strong transient complexes, a molecular trigger (i.e. phosphorylation or effector molecule) drives the equilibrium through a large conformational change between a high affinity state (low or sub nanomolar $\mathrm{K}_{\mathrm{D}}$ ) and a low affinity state (micromolar $\mathrm{K}_{\mathrm{D}}$ ). Weak transient complexes have instead micromolar dissociation constants and are characterised by a dynamic equilibrium where interaction is formed and broken continuously; their interfaces are small (mean interface for weak transient complexes was determined in $740 \AA^{2}$ by Nooren and Thornton [37] and in $810 \AA^{2}$ by Dey and co-workers [34]). Given those data, with a $\mathrm{K}_{\mathrm{D}} \approx 4 \mathrm{nM}$ and an interface area of $1086 \AA^{2}, \mathrm{CK} 2$ cannot be considered a weak transient complex. It is however impossible to distinguish between permanent and strong transient complexes solely on the basis of the dissociation constant and the interface paramenters [34]. Despite that, since no molecular triggers able to alter the affinity of the CK2 protomers have been identified so far and no significant structural rearrangements of the individual subunits are observed during the formation of the CK2 holoenzyme complex, the $\alpha_{2} \beta_{2}$ tetramer should be considered a permanent complex, until proven otherwise.

Indeed isolated $\alpha$ and $\beta$ subunits have been observed in cells [38]. However, as underlined above, crystallographic data, the $\mathrm{K}_{\mathrm{D}}$ and the stability of the enzyme in vitro strongly argue against the possibility that the CK2 $\alpha_{2} \beta_{2}$ holoenzyme can spontaneously dissociate in solution. Instead, the existence of "free", isolated $\alpha$ and $\beta_{2}$ subunits can be associated to different factors such as unbalanced expression, differential localization or transport mechanism and to competitive binding with other partners, like the A-Raf, c-Mos and Chk1 kinases all reported to interact with CK2 $\beta$ possibly using the same interface explored by CK2 $\alpha$ [39]. As already stated, molecular triggers 
capable to dissociate the complex have not been identified so far. We also excluded by crystallography that this molecular switch could reside in the phosphorylation of the CK2 $\alpha$ terminal tail (phosphorylated by Cdk1 at mitosis [40]).

\section{Working model of CK2 regulation by autoinhibitory oligomerization}

The overall regulatory model emerging from structural studies and in accordance with many biochemical, biophysical and activity data, is depicted in Figure 5. When co-localised in time and space, CK2 $\alpha$ and CK2 $\beta$-dimer spontaneously assemble into an active, symmetric, and permanent complex with a $\mathrm{K}_{\mathrm{D}} \approx 4 \mathrm{nM}$. Enzymatic activity is turned on/off by the equilibrium between the active tetramers and the inactive asymmetric trimers of tetramers that can further polymerize in higher supramolecular assemblies. These represent the latent form of the kinase in accordance with the observation that most of CK2 in crude liver extract is inactive [41]. As for the majority of protein kinases, activity is restored only upon necessity, in this case by a depolymerization process generating active free tetramers. This process can be triggered by different events like $\beta$ dephosphorylation, physical-chemical variations of the environment ( $\mathrm{pH}$, ionic strength, etc.) and/or action of substrates themselves or other effectors (highly charged macromolecules or lowmolecular-weight compounds). When the stimuli end, CK2 tetramers are assembled back in trimers and then in fibrous polymers, with the turning off of the catalytic activity. The $\beta$ subunit is fundamental in this process driving both the assembly in trimers of tetramers (via the acidic loop) and in filaments (via its C-terminus), fully deserving the name of "regulatory" subunit.

\section{Acknowledgments}

This work was supported by Italian Miur (PRIN 2008, R.B.), by FEBS (Distinguished Young Investigator Award, G.L.), and by the University of Padua (Progetto Giovani GRIC101044, G.L.). 


\section{References}

1) Niefind K, Guerra B, Ermakowa I, Issinger OG (2001) Crystal structure of human protein kinase CK2: insights into basic properties of the CK2 holoenzyme. EMBO J 20:5320-5331

2) Niefind K, Guerra B, Pinna LA et al (1998) Crystal structure of the catalytic subunit of protein kinase CK2 from Zea mays at 2.1 A resolution. EMBO J 17:2451-2462

3) Chantalat L, Leroy D, Filhol O et al (1999) Crystal structure of the human protein kinase CK2 regulatory subunit reveals its zinc finger-mediated dimerization. EMBO J 18:29302940

4) Boldyreff B, Meggio F, Pinna LA, Issinger OG (1993) Reconstitution of normal and hyperactivated forms of casein kinase- 2 by variably mutated beta-subunits. Biochemistry $32: 12672-12677$

5) Niefind K, Issinger OG (2005) Primary and secondary interactions between CK2alpha and CK2beta lead to ring-like structures in the crystals of the CK2 holoenzyme. Mol Cell Biochem 274:3-14

6) Sarno S, Vaglio P, Marin O et al (1997) Basic residues in the 74-83 and 191-198 segments of protein kinase CK2 catalytic subunit are implicated in negative but not in positive regulation by the beta-subunit. Eur J Biochem 248:290-295

7) Hathaway GM, Traugh JA (1984) Kinetics of activation of casein kinase II by polyamines and reversal of 2,3-bisphosphoglycerate inhibition. J Biol Chem. 259:7011-7015

8) Mamrack MD (1989) Stimulation of enzymatic activity in filament preparations of casein kinase II by polylysine, melittin, and spermine. Mol Cell Biochem 85:147-157

9) Leroy D, Heriché JK, Filhol O et al (1997) Binding of polyamines to an autonomous domain of the regulatory subunit of protein kinase CK2 induces a conformational change in the holoenzyme. A proposed role for the kinase stimulation. J Biol Chem 272:20820-20827 
10) Grankowski N, Boldyreff $\mathrm{B}$, Issinger OG (1991) Isolation and characterization of recombinant human casein kinase II subunits alpha and beta from bacteria. Eur J Biochem $198: 25-30$

11) Hathaway GM, Traugh JA (1979) Cyclic nucleotide-independent protein kinases from rabbit reticulocytes. Purification of casein kinases. J Biol Chem 254:762-768

12) Glover CV (1986) A filamentous form of Drosophila casein kinase II. J Biol Chem 261:14349-14354

13) Valero E, De Bonis S, Filhol O et al (1995) Quaternary structure of casein kinase 2. Characterization of multiple oligomeric states and relation with its catalytic activity. $\mathrm{J}$ Biol Chem 270:8345-8352

14) Lolli G, Pinna LA, Battistutta R (2012) Structural determinants of protein kinase CK2 regulation by autoinhibitory polymerization. ACS Chem Biol 7:1158-1163

15) Laudet B, Barette C, Dulery V et al (2007) Structure-based design of small peptide inhibitors of protein kinase CK2 subunit interaction. Biochem J 408:363-373

16) Carugo O, Argos P (1997) Protein-protein crystal-packing contacts. Protein Sci 6:22612263

17) Theis-Febvre $\mathrm{N}$, Martel $\mathrm{V}$, Laudet $\mathrm{B}$ et al (2005) Highlighting protein kinase CK2 movement in living cells. Mol Cell Biochem 274:15-22

18) Gotz C, Kartarius S, Schetting S, Montenarh M (2005) Immunologically defined subclasses of the protein kinase CK2 beta-subunit in prostate carcinoma cell lines. Mol Cell Biochem 274:181-187

19) Kristensen LP, Larsen MR, Hojrup P et al (2004) Phosphorylation of the regulatory betasubunit of protein kinase CK2 by checkpoint kinase Chk1: identification of the in vitro CK2beta phosphorylation site. FEBS Lett 569:217-223

20) French AC, Luscher B, Litchfield DW (2007) Development of a stabilized form of the regulatory CK2beta subunit that inhibits cell proliferation. J Biol Chem 282:29667-29677 
21) Litchfield DW, Lozeman FJ, Cicirelli MF et al (1991) Phosphorylation of the beta subunit of casein kinase II in human A431 cells. Identification of the autophosphorylation site and a site phosphorylated by p34cdc2. J Biol Chem 266:20380-20389

22) Pagano MA, Sarno S, Poletto G et al (2005) Autophosphorylation at the regulatory beta subunit reflects the supramolecular organization of protein kinase CK2. Mol Cell Biochem $274: 23-29$

23) Boldyreff B, Meggio F, Pinna LA, Issinger OG (1994) Efficient autophosphorylation and phosphorylation of the beta-subunit by casein kinase- 2 require the integrity of an acidic cluster 50 residues downstream from the phosphoacceptor site. J Biol Chem 269:4827-4831

24) Meggio F, Pinna LA (1984) Subunit structure and autophosphorylation mechanism of casein kinase-TS (type-2) from rat liver cytosol. Eur J Biochem 145:593-599

25) Olsen BB, Boldyreff B, Niefind K, Issinger OG (2006) Purification and characterization of the CK2alpha'-based holoenzyme, an isozyme of CK2alpha: a comparative analysis. Protein Expr Purif 47:651-661

26) Zhang C, Vilk G, Canton DA, Litchfield DW (2002) Phosphorylation regulates the stability of the regulatory CK2beta subunit. Oncogene 21:3754-3764

27) Agostinis P, Goris J, Pinna LA, Merlevede W (1987) Regulation of casein kinase 2 by phosphorylation/dephosphorylation. Biochem J 248:785-789

28) Lolli G, Ranchio A, Battistutta R. (2014) Active form of the protein kinase CK2 $\alpha_{2} \beta_{2}$ holoenzyme is a strong complex with symmetric architecture. ACS Chem Biol 9:366-371

29) Schnitzler A, Olsen BB, Issinger OG, Niefind K (2014) The protein kinase CK2(Andante) holoenzyme structure supports proposed models of autoregulation and transautophosphorylation. J Mol Biol 426:1871-1882

30) Poole A, Poore T, Bandhakavi S et al (2005) A global view of CK2 function and regulation. Mol Cell Biochem 274:163-170 
31) Raaf J, Guerra B, Neundorf I et al (2013) First structure of protein kinase CK2 catalytic subunit with an effective CK2 $\beta$-competitive ligand. ACS Chem Biol 8:901-907

32) Raaf J, Bischoff $N$, Klopffleisch $K$ et al (2010) Interaction between $C K 2 \alpha$ and $C K 2 \beta$, the subunits of protein kinase CK2: thermodynamic contributions of key residues on the CK2 $\alpha$ surface. Biochemistry 50:512-522

33) Krissinel E, Henrick K (2007) Inference of macromolecular assemblies from crystalline state. J Mol Biol 372:774-797

34) Dey S, Pal A, Chakrabarti P, Janin J (2010) The subunit interfaces of weakly associated homodimeric proteins. J Mol Biol 398:146-160

35) Nooren IM, Thornton JM (2003) Diversity of protein-protein interactions. EMBO J 22:3486-3492.

36) Perkins JR, Diboun I, Dessailly BH et al (2010) Transient protein-protein interactions: structural, functional, and network properties. Structure 18:1233-1243

37) Nooren IM, Thornton JM (2003) Structural characterisation and functional significance of transient protein-protein interactions. J Mol Biol 325:991-1018

38) Filhol O, Nueda A, Martel $\mathrm{V}$ et al (2003) Live-cell fluorescence imaging reveals the dynamics of protein kinase CK2 individual subunits. Mol Cell Biol 23:975-987

39) Filhol O, Martiel JL, Cochet C (2004) Protein kinase CK2: a new view of an old molecular complex. EMBO Rep 5:351-355

40) St-Denis NA, Derksen DR, Litchfield DW (2009) Evidence for regulation of mitotic progression through temporal phosphorylation and dephosphorylation of CK2alpha. Mol Cell Biol 29:2068-2081

41) Baggio B, Pinna LA, Moret V, Siliprandi N (1970) A simple procedure for the purification of rat liver phosvitin kinase. Biochim Biophys Acta 212:515-517 
Table 1. $\alpha / \beta$ interface parameters in symmetric $\alpha_{2} \beta_{2}$ holoenzyme

Adapted with permission from [28]. Copyright (2014) American Chemical Society.

\begin{tabular}{lrl}
\hline Parameter & Value & Comment \\
Mean Interface Area & $1086 \AA^{2}$ & $740-810 \AA^{2}$ for weak transient complexes \\
$\mathrm{f}_{\text {np }}$ (non polar fraction of interface area) & $70.5 \%$ & Largely hydrophobic \\
P-value (PISA solvation energy gain) & 0.109 & Largely hydrophobic \\
$\mathrm{R}_{\mathrm{p}}$ (residue propensity score) & 9.8 & Typical of “strong” complexes \\
$\mathrm{f}_{\text {bu }}$ (fraction of fully buried atoms) & $30 \%$ & High shape complementarity \\
$\mathrm{S}_{\mathrm{c}}$ (shape complementarity score) & 0.68 & High shape complementarity \\
$\mathrm{I}_{\text {gap }}$ (gap volume index) & 1.6 & High shape complementarity \\
$\mathrm{L}_{\mathrm{D}}$ (atomic density index) & 38.7 & High shape complementarity \\
\hline
\end{tabular}




\section{Figure legends}

Figure 1. Structure of the CK2 holoenzyme (PDB: 1JWH) [1]. a) The CK2 holoenzyme has a butterfly shape with the two catalytic $\alpha$-subunits not in contact between them. The CK2 $\beta$ tails have a fundamental role in the holoenzyme both by strengthening the $\beta$-dimer and by largely contributing to the $\alpha / \beta$ interface. The holoenzyme is asymmetric with a relative rotation of $16.4^{\circ}$ for the two halves of the tetramer. b) The asymmetric assembly generates two different $\alpha / \beta$ interfaces. Adapted with permission from [28]. Copyright (2014) American Chemical Society. c) The holoenzyme assembly in circular trimers of tetramers has been identified in the crystallographic packing [5]. The core of the interaction is between the $\beta$ acidic loop and the $\alpha p+1$ loop at the entrance of the catalytic site.

Figure 2. Details of the $\alpha / \beta$ interaction in the improved structure of the CK2 holoenzyme (4DGL) [14]. a) In 4DGL, $\beta$-Phe190 (salmon) inserts into a deep hydrophobic pocket on the CK2 $\alpha$ surface (green), differently from the 1JWH structure (orange). Adapted with permission from [14]. Copyright (2012) American Chemical Society. b) In 4DGL, $\beta$ C-terminus was built to the last Arg215 and region 194-205 was better modelled in respect to the 1JWH structure; color code as in a). Adapted with permission from [14]. Copyright (2012) American Chemical Society. c) In the improved 4DGL structure, the two $\alpha / \beta$ interfaces are larger than in 1JWH (Fig. 1b). Adapted with permission from [28]. Copyright (2014) American Chemical Society.

Figure 3. Oligomeric organization in the 4DGL structure. Adapted with permission from [14]. Copyright (2012) American Chemical Society. a) Different inter-tetrameric contacts can be identified. The interaction between the $\beta$ acidic loop ( $\beta$ subunit in cyan with its acidic loop in yellow) and the $\alpha \mathrm{P}+1$ loop ( $\alpha$ subunit in orange with its $\mathrm{P}+1$ loop in green) is responsible for the assembly in circular trimers of tetramers. The interaction between the $\alpha \mathrm{G}$ and the $\mathrm{C}$-terminal tail of 
a different $\beta$ subunit (purple) and the $\alpha \mathrm{C}$-terminal lobe and active site (orange) is responsible for a piling organization of trimers of tetramers. Position of the first amino acid visible at the $\beta$ Nterminus (Val7) is compatible with the autophosphorylation of the upstream residues Ser2 and Ser3. Phosphorylated residues could then interact with the $\alpha$ basic cluster (red) b) Insertion of the $\beta$ Cterminus into the $\alpha$ active site disrupts the correct orientation of $\alpha$ residues involved in nucleotide binding. c) In the filamentous organization, $\mathrm{C}$-termini of three $\beta$ subunits are plugged into the following trimer.

Figure 4. Structural features of the symmetric tetrameric holoenzyme. Adapted with permission from [28]. Copyright (2014) American Chemical Society. a) Structure of symmetric tetramer in salmon (4MD7) [28] superposed to the asymmetric 4DGL tetramer (cyan). b) In 4DGL the assembly of three tetramers (green, cyan and purple) in trimers of tetramers induces the distortion not present in the symmetric tetramer (salmon). c) In the symmetric tetramer, the two $\alpha / \beta$ interfaces are very similar in size and nature.

Figure 5. Proposed model of CK2 regulation. Reprinted with permission from [28]. Copyright (2014) American Chemical Society. CK2 $\alpha$ and $\beta$ subunits readily assemble into active and symmetric tetramers. Activity is turned off through organization in inactive trimers and filaments. Activity is restored upon a depolymerazion process induced by variations in the physical-chemical environment, action of substrates or effectors, or other events. 